\title{
Reverse-phase protein microarrays (RPPA) as a diagnostic and therapeutic guide in multidrug resistant leukemia
}

\author{
TULLIA MARALDI $^{1}$, JESSIKA BERTACCHINI $^{1,2}$, MARTA BENINCASA ${ }^{1}$, MARIANNA GUIDA $^{1,2}$, \\ ANTO DE POL ${ }^{1}$, LANCE A. LIOTTA ${ }^{4}$, EMANUEL PETRICOIN III ${ }^{4}$, \\ LUCIO COCCO $^{2}$ and SANDRA MARMIROLI ${ }^{1,3}$
}

\begin{abstract}
${ }^{1}$ Department of Anatomy and Histology and CIPro Proteomics Centre, University of Modena and Reggio Emilia, Via Del Pozzo 71, I-41100 Modena; ${ }^{2}$ Department of Anatomical Sciences, Cellular Signalling Laboratory, Via Irnerio 48, I-40126 Bologna; ${ }^{3}$ I.G.M.-CNR, Unit of Bologna, Laboratory of Cellular Biology and Electron Microscopy c/o IOR, Via di Barbiano, I-40125 Bologna, Italy; ${ }^{4}$ Center for Applied Proteomics \& Molecular Medicine, GMU, Fairfax, VA, USA
\end{abstract}

Received August 9, 2010; Accepted September 20, 2010

DOI: $10.3892 /$ ijo. 2010.850

\begin{abstract}
Reverse-phase microarray assays using phosphospecific antibodies (RPPA) can directly measure levels of phosphorylated protein isoforms. In the current study, lysates from parental and multidrug resistant (MDR) CEM leukemia cells were spotted onto reverse-phase protein microarrays and probed with a panel of phospho-antibodies to ERK, PCK and Akt pathways. In particular, the Akt pathway is considered to play significant roles in leukemia and Akt inhibitor therapy has been proposed as a potential tool in the treatment of this disease. The RPPA data prompted us to investigate deeper this pathway. Here, we found that whereas total Akt1 protein level is higher in parental CEM cells, the activated isoform content, p-Akt1, increases in doxorubicin-selected CEM cells (MDR-CEM). This was backed up by Western blot analysis, confirming that Akt1 activity/phosphorylation may be upregulated in MDR-CEM cells. Further exploration of inhibitory therapy in this system was evaluated. The TNF-related apoptosis-inducing ligand, TRAIL, has been shown to selectively kill tumor cells. Herein, we describe that in MDRCEM cells TRAIL responsiveness correlates with a reduced expression of endogenous Akt1, suggesting that the MDR phenotype associated to P-gp sensitizes cells to TRAIL therapy.
\end{abstract}

\section{Introduction}

Drug resistance is the major reason for the ultimate failure of chemotherapy in the majority of human cancers. Certain types of cancer display intrinsic resistance to multiple chemotherapeutic drugs while many other cancers acquire multidrug resistance (MDR) during chemotherapy. MDR is frequently

Correspondence to: Dr Sandra Marmiroli, Department of Anatomy and Histology, University of Modena and Reggio Emilia, Via Del Pozzo 71, I-41100 Modena, Italy

E-mail: marmiroli.sandra@unimore.it

Key words: RPPA, MDR, TRAIL, Akt/PKB, phosphorylation associated with the overexpression of P-glycoprotein (P-gp), a $170 \mathrm{kDa}$ ATP-dependent transmembrane protein encoded by the MDR1 gene. P-gp resides in lipid raft microdomains (1) and is capable of pumping a number of structurally unrelated chemotherapy drugs and other compounds out of the cell.

The susceptibility of cancer cells to apoptosis induced by chemotherapeutic drugs depends on the balance between important pro-apoptotic and anti-apoptotic (survival) signals. ERK (extracellular-signal regulated kinases) and PI3K (phosphatidylinositol 3-kinase)/Akt kinase cascades play key roles in regulation of apoptotic responses and are known as important survival cell signaling pathways.

Many studies demonstrated the ability of modulators of protein phosphorylation/dephosphorylation to control the P-gp mediated multidrug resistance $(2,3)$. It is known that P-gp can serve as a substrate for several protein kinases, such as protein kinase $\mathrm{C}(\mathrm{PKC})$, protein kinase $\mathrm{A}$ (PKA), CKII, P-gp specific kinase, but the functional significance of its phosphorylation is not yet clear and some reports indicate that certain protein kinase blockers inhibit drug transport by mechanism independent of P-gp phosphorylation $(4,5)$. However, in several cancer cells a parallel expression of P-gp and activation and/or expression of mitogen-activated protein kinases (MAPKs), PKC, Akt kinase was found. Moreover, these protein kinase pathways have been reported to modulate the expression or activity of P-gp in some multidrug resistant cell lines (6-8). In particular, it has been shown that the reversal of the MDR phenotype can probably occur by the suppression of P-gp expression via the inhibition of the PI3K/Akt/NF- $\mathrm{kB}$ signaling pathway (9).

AKT, a serine/threonine protein kinase and a key downstream mediator of the phosphatidylinositol 3-kinase pathway, has been well studied for its role in cell growth, survival, migration, and differentiation both in physiological and pathological conditions. Three forms of AKT (AKT1, AKT2, and AKT3) exist in mammalian cells. Whereas they are known to share common substrates and functions, their specific role and task is still an intriguing and challenging question (10).

Many cellular proteins are involved in regulating apoptosis, including members of the tumor necrosis factor (TNF) receptor 
superfamily of death receptors such as TNF- $\alpha$ receptor 1 , Fas, and the tumor necrosis factor related apoptosis-inducing ligand (TRAIL) receptors DR4 and DR5 (11). When productively bound by their respective ligands (TNF- $\alpha$, FasL, and TRAIL) on the cell surface, the death receptors trimerize and a caspasedependent apoptotic pathway is initiated (12-15). TRAIL binds to four membrane receptors including death receptor 4 (DR4, TRAIL-R1), KILLER/DR5 (TRAIL-R2, TRICK2), DcR1 (TRID, TRAIL-R3), DcR2 (TRUNDD or TRAIL-R4), and a soluble receptor, osteoprotegerin (OPG). Only DR4 and DR5 contain cytoplasmic death domains and mediate apoptosis upon binding to TRAIL in sensitive target cells (16). In contrast, neither DcR1 nor DcR2, which contains a truncated cytoplasmic death domain, mediate apoptosis after binding to TRAIL; thus serving as decoy receptors to sequester TRAIL at the cell surface (16). TRAIL is a very promising cytokine for anticancer therapy since cancer cells are more sensitive to its cytotoxic activity compared to normal cells. However, despite early promising results, recent studies have identified several TRAIL-resistant cancer cell lines (17-19). Several lines of evidence support a role for P-gp in enhancing TRAILinduced apoptosis: in fact, TRAIL induced preferential apoptosis in P-gp-bearing cells selected for resistance to several anticancer agents, as well as in cells transfected with the MDR1 and overexpressing P-gp (20).

Leukemia cell lines can develop resistance to TRAILinduced apoptosis, and this can be due to increased level of pAkt which is partly due to phosphorylation of PTEN (21). In particular, the doxorubicin-treated human T-lymphoblastic leukemic CEM cell line is characterized by both expression of P-gp and high levels of Akt activation (22-24) and parental CEM cells are more resistant to TRAIL-induced apoptosis, compared with other human chronic myeloid leukemic cell lines (25).

Although leukemia drug resistance has been defined by means of gene expression profiling, at a proteomic level data are controversial as to how signaling pathways are differentially activated and serve to control cell proliferation and drug resistance regulation. Given these premises, we decided to perform a comparative analysis of the endogenous phosphorylome in two isogenic variants of human $\mathrm{T}$ lymphoblastoid CEM cell line, one sensitive to drug-induced apoptosis, while the other, referred to as MDR-CEM, derived from the parental one by selection with vinblastine treatment, and displaying a cross-resistance to many other chemotherapeutic drugs $(26,27)$. In order to compare the phosphorylation profile of multiple proteins, we utilized the high throughput reversephase protein array technology (RPPA). Our results indicate that the two subtypes MDR-CEM and CEM showed distinct pathway activation that are not obvious from previous gene expression studies. We also show that basal levels of phosphorylation of key signaling nodes may have diagnostic utility in predicting response to selective inhibitors of Akt pathway. In an attempt to assess Akt1 as a candidate for treatment of MDR malignancies, parental CEM leukemia cells and the isogenic MDR-CEM subline were screened for TRAIL responsiveness. The results presented here confirmed that TRAIL was completely ineffective on parental CEM cells, while triggered massive apoptosis in the MDR-counterpart. This pattern correlates with the opposite expression and activation trend of Akt1 in MDR-CEM cells. Furthermore, in these cells TRAIL treatment affects Akt1 protein level, rather than its activation, through a degradation mechanism. This interesting result prompted us to analyze deeper the effect triggered by TRAIL on the above phosphorylation pathways.

\section{Materials and methods}

Materials. Doxorubicin chloridrate was from Pharma Italia; MG-132, anti-actin and cycloheximide were from Sigma (St. Louis, MO, USA); the broad caspase inhibitor Z-Vad fmk and anti-MDR were from Calbiochem (La Jolla, CA, USA). TRAIL was from PeproTech (London, UK). Anti-PHLPP was from Bethyl Lab. (Montgomery, TX, USA).

The following antibodies were used: anti-Akt1, anti-Akt2, anti-NF-кB, anti-PARP, all from Santa Cruz Biotechnology, CA; anti-phospho-GSK3ß, antiGSK3ß, anti-phosphoAkt(Ser473), anti-phospho-Akt(Thr308), anti-phospho-PDK1, anti-phospho-PTEN, anti-PTEN, anti-phospho-mTOR, anti-mTOR and anti-phospho-PRAS40 from Cell Signaling Technology (Beverly, MA USA). Horseradish peroxidaseconjugated (HRP) secondary antibodies as well as chemiluminescence (ECL) reagents were from Amersham Pharmacia Biotech (Piscataway, NJ, USA). All electrophoresis reagents, nitrocellulose and protein assay kits were from Bio-Rad (Hercules, CA, USA). All other chemicals were of the highest quality available.

Cell culture and treatments. The human T-lymphoblastic leukemic CEM cell line was maintained in RPMI-1640 medium (EuroClone Ltd., UK) supplemented with $10 \%$ fetal bovine serum (FBS, EuroClone Ltd., UK). The multidrug resistant subline, MDR-CEM, was obtained by continuous exposure to doxorubicin, as described by Cenni et al (28) and maintained as parental-U2OS. Cells were treated with $0.5-1 \mu \mathrm{g} / \mathrm{ml}$ TRAIL or vehicle alone and used for experiments. Where indicated, cells were pre-treated with $20 \mu \mathrm{M}$ cycloheximide, $10 \mu \mathrm{M}$ MG-132 or $20 \mu \mathrm{M} \mathrm{Z-Vad} \mathrm{fmk.}$

Preparation of cell extracts. CEM cells were extracted by addition of RIPA buffer (20 mM Tris-Cl, pH 7.0, 1\% NP-40, $150 \mathrm{mM} \mathrm{NaCl}, 10 \%$ glycerol, $10 \mathrm{mM}$ EDTA, $20 \mathrm{mM} \mathrm{NaF}$, $5 \mathrm{mM}$ sodium pyrophosphate, $1 \mathrm{mM} \mathrm{Na}_{3} \mathrm{VO}_{4}$, and freshly added Sigma-Aldrich Protease Inhibitor Cocktail) at $4^{\circ} \mathrm{C}$ for $10 \mathrm{~min}$. Lysates were cleared by centrifugation and used for immunoprecipitation experiments, as described below. Total lysate $(50 \mu 1)$ was boiled in SDS sample buffer, resolved, and immunoblotted with the indicated antibodies. In brief, samples were loaded onto an SDS-polyacrylamide gel, blotted on Immobilon-P membranes (Millipore, MA), and processed by Western blotting with the indicated antibody, detected by Supersignal substrate chemiluminescence detection kit (Pierce, UK). Quantitation of the signal was obtained by chemiluminescence detection on a Kodak Image Station 440CF and analysis with Kodak 1D Image software.

Flow cytometry. The expression of TRAIL receptors and P-gp was analyzed by indirect staining using mouse anti-human TRAIL-R1, TRAIL-R2 (Alexis, Lausen, Switzerland) and mouse anti-human P-gp (BD Pharmingen) as specified by 
Milani et al (29) followed by phycoerythrin-conjugated rabbit anti-mouse IgG (Sigma). Non-specific fluorescence was assessed by using normal mouse IgG followed by the second antibody as described above. Analyses were performed with a FACScan flow cytometer and Lysis II software (both from Becton-Dickinson, San Jose, CA, USA).

Reverse-phase protein arrays. Cells were grown in standard media and $10 \%$ fetal bovine serum until they reached $60-80 \%$ confluence and then lysed directly in plates in buffer consisting of a $2.5 \%$ solution of 2-mercaptoethanol in loading buffer/TPER plus phosphatase and protease inhibitors. All samples were diluted to a final concentration of $0.5 \mathrm{mg} / \mathrm{ml}$ and then $30 \mu 1$ of each sample, arrayed in a series of 6-fold dilutions, was printed in duplicate on slides. Lysates derived from HeLa cells or HeLa cells treated with pervanadate were also printed on each slide as low and high phosphorylation controls, respectively. The slides were then subjected to immunostaining with a panel of 90 commercially available antibodies primarily directed against specific phosphorylated or cleaved proteins, including PI-3K/Akt, ERK/MAPK, PKCs and caspase-dependent apoptosis. Each of these antibodies had previously undergone extensive validation for both phosphorylation and protein specificity using single band detection at the appropriate MW by Western blotting. To estimate the total protein amount, selected arrays were stained with Sypro Ruby protein blot stain (Molecular Probes, Eugene, OR, USA) and visualized on a Fluorchemk imaging system (Alpha Innotech, San Leandro, CA, USA). Slides were stained on an automated slide stainer (Dako, Carpinteria, CA, USA) using a biotin-linked peroxidase catalyzed signal amplification. Finally, the primary antibodies at concentrations ranging from 1:50 to $1: 1000$ were applied for $30 \mathrm{~min}$ followed by the secondary link antibody for $30 \mathrm{~min}$ (concentration 1:10 for anti-mouse antibodies and 1:5000 for anti-rabbit antibodies). To allow normalization of total protein on printed arrays, one to two slides in each print run were stained with Sypro Ruby protein blot stain (Invitrogen) and the value of these stained arrays used for normalization of all end-point values.

The intensity value for each end point was determined by identifying spots for each duplicate dilution curve for each sample that were within the linear dynamic range of the staining after background subtraction with each spot (within slide local background and also against a slide stained with secondary antibody only). Single intensity values were obtained by multiplying each spot in the linear range by its dilution factor and averaging candidate linear points. Finally, each value was normalized relative to the total protein intensity value for that sample derived from the Sypro Ruby-stained slide.

Statistical analysis. Statistical analysis was performed with Student's unpaired t-test. Results are the mean of at least three independent experiments. Differences were considered significant at $\mathrm{P}<0.05$.

\section{Results}

$P$-gp expression in CEM cells. The multidrug resistant clone, herein called MDR-CEM, has been selected by continuous
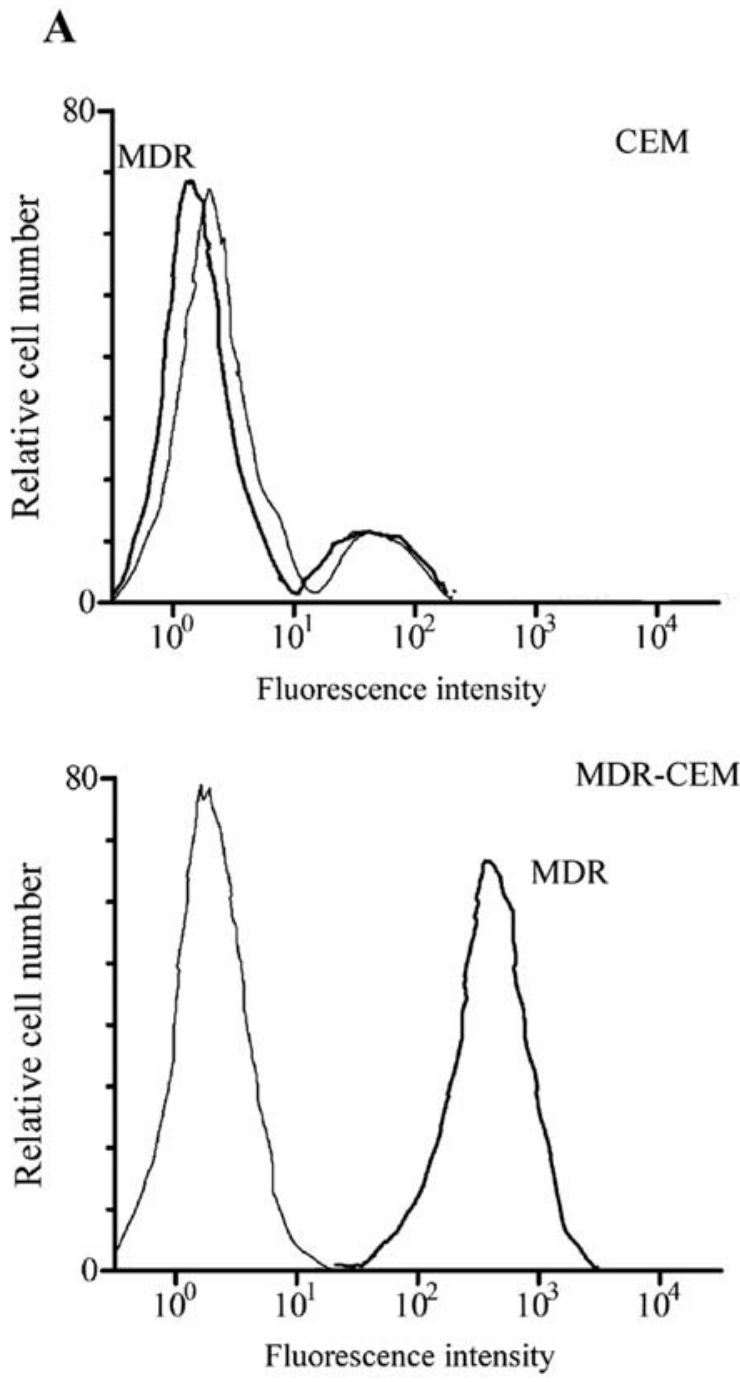

B

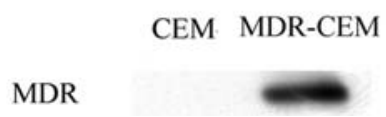

Figure 1. Surface expression of MDR-1 gene in parental and MDR-CEM cells by cytometric and Western blot analysis. (A) Gray histograms represents MDR fluorescence, whereas white histograms are the background fluorescence. A representative of three separate experiments is shown. (B) Representative immunoblotting showing MDR expression level in CEM cells treated or not with doxorubicin as reported in Materials and methods. Forty micrograms of protein per lane were electrophoresed and immunoblotted. The data shown are representative of three independent experiments.

exposure of CEM cells to doxorubicin and characterized (27). To investigate the surface expression of P-gp, parental- and MDR-CEM cells were fixed and incubated with a FITCconjugated anti-P-gp antibody and subjected to flow cytometric analysis (Fig. 1A). The results show that, while parental CEM is completely P-gp deficient, MDR-CEM cells possess considerable levels of the membrane transporter, as confirmed also by Western blot analysis (Fig. 1B).

RPPA analysis of the phosphorylation pathways activated in $M D R-C E M$ cells. With the reverse-phase protein microarray (RPPA) platform, the entire cellular proteome is immobilized 
A
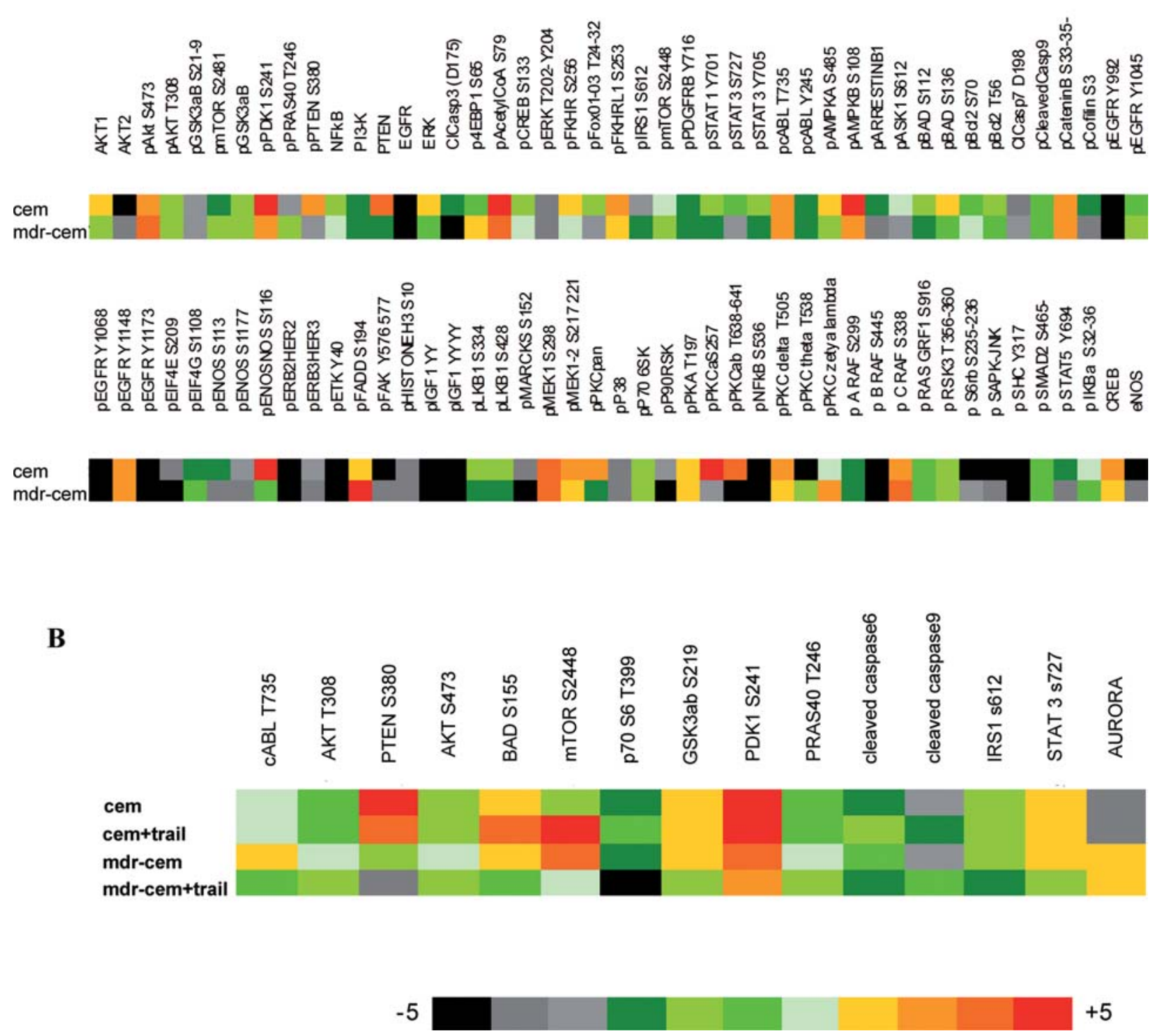

Figure 2. Heat maps of RPPA analysis of parental and MDR-CEM cells. (A) Heat map representation of RPPA analysis showing parental and MDR-CEM cells subjected to immunostaining with a panel of 90 commercially available antibodies. The color in each cell reflects the level of expression. (B) Heat map representation of RPPA analysis showing parental and MDR-CEM cells, treated or not with for $16 \mathrm{~h}$ TRAIL, subjected to immunostaining with a panel of 30 commercially available antibodies. The color in each cell reflects the level of expression.

on a nitrocellulose covered glass substratum, allowing subsequent immunodetection both of the total levels and of phosphorylated, or activated, signaling proteins. We profiled protein lysates from parental- and MDR-CEM cells with a panel of 90 antibodies recognizing primarily phosphorylated epitopes for ERK, PCK and Akt pathways (Fig. 2A), already validated by the lab of Liotta et al (30-32). It is interesting to note that several endpoints are differentially expressed or activated. In particular, we address here the Akt pathway phosphorylome, and its relation to the MDR-phenotype.

Fig. 3A shows that Akt pathway activation seems to play a key role in determining the different somatic features of MDR-CEM cells. In particular, given that parental-CEM cells express higher levels of the Akt1 protein, the unexpected finding that Akt phosphorylation at S473 is higher in MDRCEM, while phosphorylation at T308 is comparable in the two cell clones, suggested that Akt1 phosphorylation at S473 may represent an important cue of MDR in CEM cells, as observed before by us and others in different cell types (28). Since Akt activity is finely tuned by well known kinases and phosphatases, we analyzed the phosphorylation profile of upstream and downstream Akt regulatory molecules in the two cell types.

In good agreement with the above result, we can observe that PTEN protein level, as well as phosphorylation, is almost undetectable in MDR-CEM cells (Fig. 3B). Likewise, the very low content of the $\mathrm{pS} 473$ protein phosphatase PHLPP in MDR-CEM cells, with respect to S-CEM, substantiate the 
A

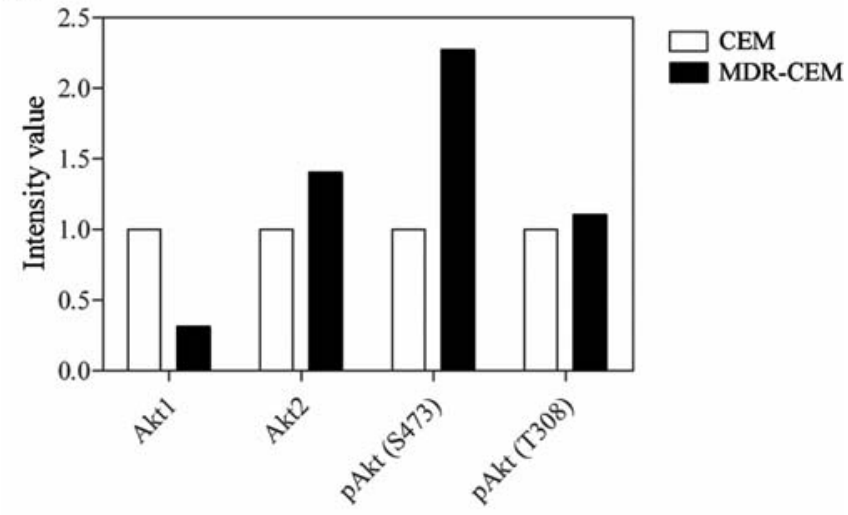

B

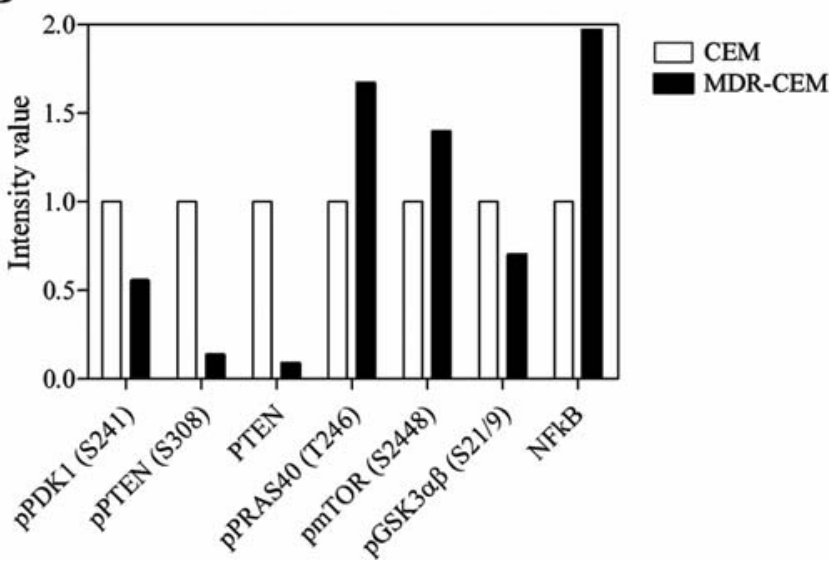

Figure 3. RPPA analysis of parental and MDR-CEM cells. RPPA analysis was performed as described in Materials and methods. Comparisons of staining intensities for end points that were statistically different between parental and MDR cell lines. The data shown are representative of two independent experiments.

above result, accounting for the much higher Akt kinase activity/phosphorylation in these cells (Fig. 4). On the other hand, the activity of the S473-kinase, mTORC2 (33), is clearly augmented in MDR-CEM cells, as detected by anti$\mathrm{pS} 2481$, contributing to explain the discrepancy between the two subclones.

The proline-rich Akt substrate of $40 \mathrm{kDa}$ (PRAS40) was identified as a novel mTOR binding partner that mediates Akt signals to mTOR and is therefore regarded as an important regulator of insulin sensitivity of the Akt-mTOR pathway. Upon phosphorylation by Akt, indeed, PRAS40 binds to 14-3-3, thus relieving its inhibition of mTORC1 (33). Our data provided evidence that PRAS40 is heavily phosphorylated in multidrug resistant cells, compared to parental cells (Fig. 3B).

As far as the Akt-downstream pathway, we investigated the epitope-specific phosphorylation of well known substrates such as GSK3ß (glycogen synthase kinase 3ß), which directly phosphorylates and thus inactivates glycogen synthase, and the transcription factor $\mathrm{NF}-\kappa \mathrm{B}$, which mediates at least to some extent Akt-promoted cell survival. Fig. 3B clearly shows that phosphorylation of p65 at Ser536 is doubled in MDR-CEM, indicating that NF- $\mathrm{\kappa B}$ transcriptional activity is much more sustained in these cells. Conversely, GSK3ß

\section{CEM MDR-CEM}

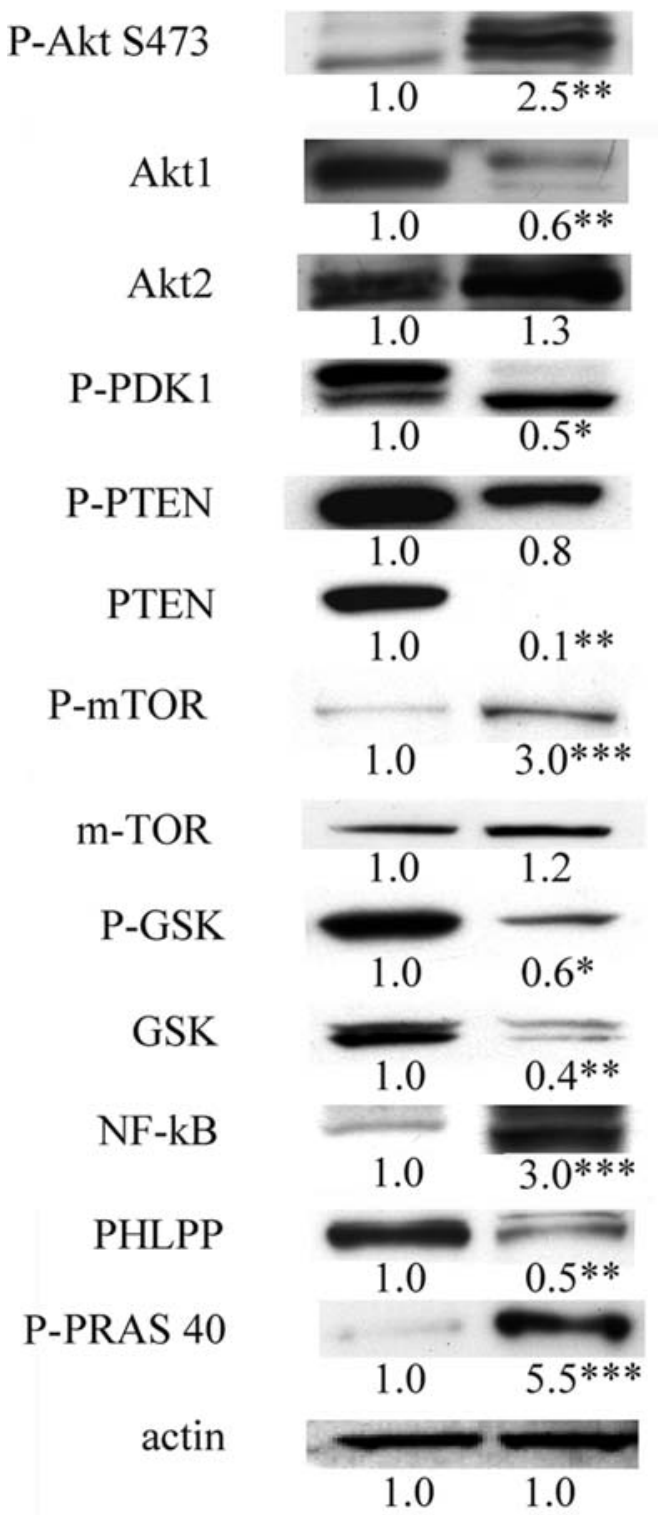

Figure 4. Western blot analysis of the Akt pathway activated in parentaland MDR-CEM cells. Samples were electrophoresed, immunoblotted and revealed for the indicated antibodies. The data shown are representative of three independent experiments. Actin was used as an internal control.

phosphorylation is similar, if not a little weaker in MDR cells than in sensitive cells. However, this result can be easily explained by the observation that the amount of total GSK3ß is more than halved in MDR-CEM cells, therefore the phosphorylation decreased accordingly (not shown).

In order to serve as both a diagnostic and a therapeutic guide, the correlation of these results with biological and clinical information has to be confirmed by Western blot analysis.

Western blot analysis of the Akt pathway activated in MDRCEM cells. Most importantly, we found a good concordance between the phosphorylation profile determined by Western blot analysis and that obtained by RPPA analysis (Fig. 4), indicating that determination of phopshorylated protein status 
A



C

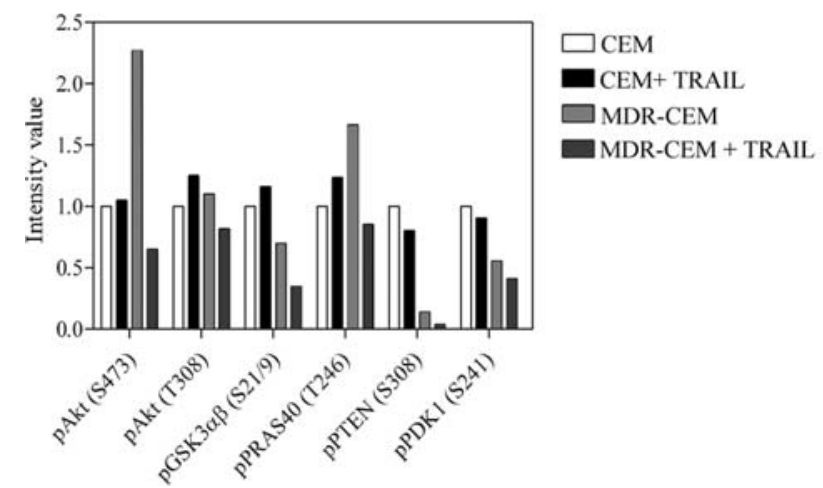

B

CEM

MDR-CEM
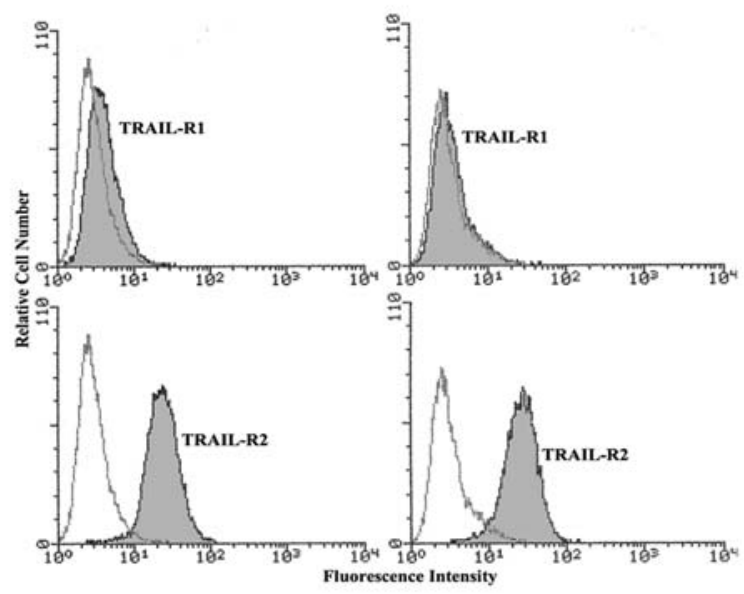

D

Figure 5. TRAIL sensitivity and TRAIL-R1 and -R2 expression in parental- and multi-drug resistant CEM cells. (A) Western blot analysis of PARP, activated (P-Akt) and total Akt expression level in parental and MDR-CEM cells treated or not for $16 \mathrm{~h}$ with TRAIL. Actin was used as an internal control. (B) Flow cytometric evaluation of surface expression of TRAIL receptors in parental and MDR-CEM cells. Gray histograms represent cells stained with antibodies specific for the indicated TRAIL receptors (TRAIL-R1 and TRAIL-R2), whereas white histograms represent the background fluorescence obtained by staining the same sample with isotype-matched control antibodies. A representative of four different experiments is shown. (C and D) RPPA analysis was performed as described in Materials and methods. Comparisons of staining intensities for end-points that were statistically different between parental and MDR cell lines. Values represent the mean of four replicates.

by RPPA in this model is reproducible. Furthermore, to deeper investigate the Akt regulation in MDR-CEM cells, we analyzed by Western blot analysis (Fig. 4) the expression of several effectors of the Akt pathway.

As we have pointed out above, the levels of $\mathrm{PH}$ domain leucine-rich repeat protein phosphatase (PHLPP), that specifically dephosphorylates the hydrophobic motif of Akt (Ser473 in Akt1) triggering apoptosis and suppressing tumor growth (35), are markedly reduced in MDR-CEM cells that have elevated Akt Ser473 phosphorylation.

The intensity of GSK is much higher in parental CEM cells, indicating that the phosphorylation pattern observed with RPPA analysis is due to the protein expression (Fig. 4). On the other hand, mTOR is equally present in both cell lines (Fig. 3), while Fig. 2B shows an increased mTOR phosphorylation in MDR-CEM cells.

Analysis of TRAIL sensitivity and TRAIL-RI and -R2 expression in parental- and multi-drug resistant CEM cells. Hyperactivation of Akt tips the balance of cells into survival pathways and is often correlated with tumor progression.
Our current study demonstrated that the acquired resistance to doxorubicin was partly due to activation of Akt1 and inactivation of PTEN and PLHPP.

We therefore investigated the response of both parental and resistant CEM cells to TRAIL, a member of the tumor necrosis factor (TNF) family of cytokines that is considered to induce death of cancer cells but not normal cells, preferentially causes apoptosis through the same mechanisms, such as increased level of pAkt and loss of PTEN activity (21). Cells were treated with doses of TRAIL $\leq 1 \mu \mathrm{g} / \mathrm{ml}$ for $16 \mathrm{~h}$, collected, and subjected to Western blotting or PI staining and FACS analysis.

As assessed by WB detection of cleaved poly(ADP-ribose) polymerase (PARP) (Fig. 5A), while untreated cells have similar PARP levels, only MDR-CEM cells exhibit a marked responsiveness to TRAIL-induced apoptosis. However, Fig. 5B shows the profile of TRAIL receptors obtained by FACS: the parental- and MDR-CEM cells express comparable levels of TRAIL-R1 and -R2, hampering any correlation with resistance or sensitivity to TRAIL-induced apoptosis and suggesting significant clinical implications for the use of TRAIL in treating neoplasms that have failed chemotherapy. 
Akt1 expression, unlike the activity, leads to TRAIL responsiveness of MDR-CEM cells. Since both parental- and MDRCEM cells express high levels of active TRAIL receptors, but TRAIL effectiveness is limited to MDR-CEM, we then investigated the Akt-related post-receptor intracellular mechanisms of the two cell types. To begin with, we examined the balance between total and phosphorylated Akt. RPPA test indicates that Akt phosphorylation at T308 and S473 decreases in MDR-CEM in response to the presence of TRAIL (Figs. 2B and 5C). Then the amount of total and activated Akt was analyzed by Western blotting followed by densitometric scanning, yielding a remarkable difference between parentaland MDR-CEM cells (Fig. 5A). In fact, as shown in Fig. 4, parental-CEM cells express much more Akt1 than their MDR counterpart, though considerably less active. Indeed our data show that TRAIL treatment causes a dramatic reduction of Akt1 protein level, and therefore of the protein phosphorylation (Fig. 5A), suggesting that TRAIL responsiveness is associated to Akt1 presence in MDR-CEM cells.

In particular, TRAIL treatment also modulates Akt key effectors: in particular Fig. 5D shows that phosphorylation of PRAS40, the mTOR binding partner that mediates Akt signals to mTOR, is abrogated, confirming the link between TRAIL responsiveness and $\mathrm{Akt} / \mathrm{mTOR}$ pathway. It is interesting to note that TRAIL treatment results in a negative modulation of the whole Akt pathway, as shown by the further decrease observed in the case of pPDK1, pPTEN and pGSK (Fig. 5C). The above result can be ascribed to the dramatic degree of apoptosis induced by TRAIL in this cell subclone.

Apoptosis induction by TRAIL is shown by Fig. 5D: the increase of cleaved caspases 6 and 9 clearly indicates that TRAIL treatment drives MDR-CEM cells to caspase-mediated cell death. Moreover, Bad phosphorylation, that is important in the prevention of apoptosis, is higher in untreated MDR-CEM cells.

Akt1 modulation in multi-drug resistant CEM cells exposed to TRAIL. We then sought to investigate whether the modulation of Akt1 content in MDR-CEM cells results from a reduced synthesis or an increased degradation of Akt1. As shown in Figs. 5A and 6, the long-term TRAIL stimulation strongly reduces expression of endogenous Akt1, while the phosphorylation level seems not to be crucial.

Although Akt level is quite stable in MDR-CEM cells after treatment with the inhibitor of protein biosynthesis Cycloheximide, co-treatment with TRAIL triggers Akt fast degradation. The treatment with the broad proteasome inhibitor MG-132 (MG) does not abrogate TRAIL-dependent Akt1 degradation, as shown in Fig. 6. The reduced content of endogenous Akt1 is most likely due to the activation of apical caspases, leading to Akt1 degradation, as this reduction was reversed by pre-treatment with the broad caspase inhibitor Z-Vad (Fig. 6).

\section{Discussion}

Analysis of the genome provides important information on the somatic genetic changes existing in the tissue; however, it is the proteins that do the work of the cell. Diseases such as cancer are caused by derangements in cellular protein

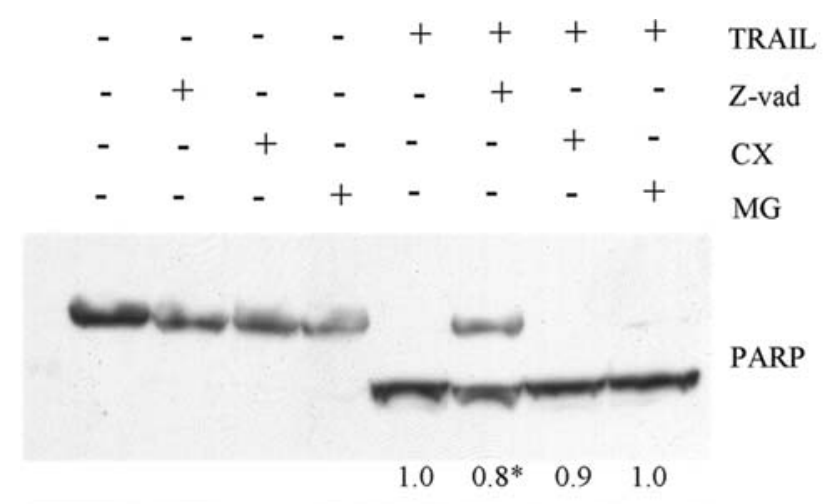

P-Akt S473

$\begin{array}{lllllll}1.0 & 0.9 & 0.8 & 0.8-1.0 & 1.9^{*} & 0.8 & 1.0\end{array}$

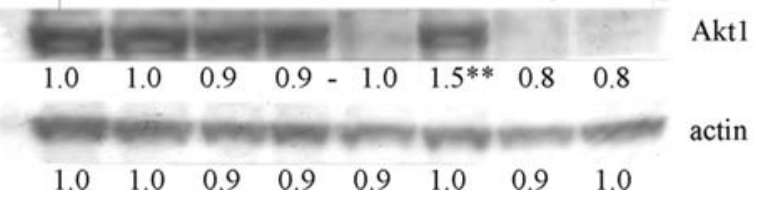

Figure 6. Akt1 modulation in multi-drug resistant CEM cells exposed to TRAIL. MDR-CEM cells, pre-treated for $16 \mathrm{~h}$ with $20 \mu \mathrm{M}$ cycloheximide or $20 \mu \mathrm{M} \mathrm{Z}$-Vad fmk and for $1 \mathrm{~h}$ with $10 \mu \mathrm{M} \mathrm{MG}-132$, were treated or not with $0.5 \mu \mathrm{g} / \mathrm{ml}$ TRAIL. Lysate samples were electrophoresed, immuno-blotted and revealed for the indicated antibodies. The data shown are representative of three independent experiments. Actin was used as an internal control.

molecular networks and cell signaling pathways. These pathways contain a large and growing collection of drug targets, governing cellular survival, proliferation, invasion, and cell death. Proteomics has the potential to provide answers in cancer pathogenesis and to direct targeted therapy through the comprehensive analysis of protein expression levels and activation status. The realization of this potential requires the development of new, rapid, high-throughput technologies for performing protein arrays on patient samples, as well as novel analytical techniques to interpret them. The clinical utility of reverse-phase protein microarrays (RPPA) lies in its ability to generate a functional map of known cell signaling networks or pathways for an individual patient (36).

In this study we used RPPA analysis to investigate the signaling pathway characteristic of a human leukemia cell line resistant to doxorubicin treatment (MDR-CEM). The drug efflux activity of P-gp represents a common mechanism that tumor cells use to escape from death induced by different chemotherapeutics agents (6). It has been found that modulators of some protein kinase pathways, especially MAPK (ERK, p38-MAPK), can influence drug transport activity of P-gp in multidrug resistant L1210/VCR, a mouse leukemic cell line $(6,37)$. Here we found that the Akt pathway is linked to multidrug resistance of CEM cells. In fact, one of the most effective antiapoptotic survival pathway in mammals is constituted of phosphoinositide 3-kinase (PI3K)/Akt $(38,39)$. The active, phopshorylated form of Akt (pAkt) promotes cell proliferation and survival by phosphorylating downstream molecules that regulate cell cycle and apoptosis (40). We demonstrate that the activated form of the isoform 1 of 
Akt (Akt1), rather than the protein content, is a typical marker of multidrug resistance. In fact, Akt1 protein level is lower in MDR-CEM cells than in parental CEM cells, indicating that the only genome analysis of leukemic patient could be misleading.

RPPA and Western blot analysis data then confirmed that S473 phosphorylation of Akt1 and the upstream and downstream transduction pathway play a pivotal role in modulating MDR-CEM survival. It has been published that there is molecular proximity between P-gp, and DR4, DR5, and DcR1 (receptors of TRAIL), then it is plausible that crosstalk between P-gp and TRAIL receptors may take place in lipid raft microdomains (20). Moreover, recent data clearly showed that lipid raft microdomains are closed chambers in which death receptors, including DR4 and DR5, as well as procaspase-8, procaspase-10, FADD, and Bid reside, and that death-mediated apoptotic signaling mechanisms are dependent upon lipid rafts (41-43). The TNF-related apoptosis-inducing ligand, TRAIL, has been shown to selectively kill tumor cells. This property has made TRAIL and agonistic antibodies against its death inducing receptors (TRAIL-R1 and TRAIL-R2) a most promising novel biotherapeutic agent for cancer therapy. The TRAIL death receptor-mediated 'extrinsic' pathway and the 'intrinsic' pathway, which is controlled by the interaction of members of the Bcl-2 family, interact with each other in the decision about life or death of a cell. Apoptotic and non-apoptotic signaling is influenced by the NF- $\mathrm{B}, \mathrm{PKB} / \mathrm{Akt}$ and the MAPK signaling pathways (44). Further understanding of the molecular mechanisms of TRAIL-induced apoptosis in drug-sensitive and -resistant cells should provide insight and approaches useful for the development of more effective therapeutic strategies to eliminate cancer cells.

An analysis of the post-receptor events showed that TRAIL responsiveness correlates with a reduced expression of endogenous Akt1. Furthermore, our results confirm the observations indicating that the upregulation of $\mathrm{P}$-gp in neoplasms that have failed chemotherapy can be exploited as an Achilles heel that sensitizes the cancer cells to TRAIL therapy.

\section{Acknowledgements}

This work was supported by the following grants: MIUR grants prin 2008THTNLC_005, FIRB RBIN04NR2S_001; FIRB RBRN07BMCT_002; ISS ONCOPROTEOMICA 527/B/3\% $/ 4$; UNIMORE, cofin azioni di mobilitá to J.B. and FAR 2008.

\section{References}

1. Bacso Z, Nagy H, Goda K, Bene L, Fenyvesi F, Matkó J and Szabó G: Raft and cytoskeleton associations of an ABC transporter: P-glycoprotein. Cytometry A 61: 105-116, 2004.

2. Ding S, Chamberlain M, McLaren A, Goh L, Duncan I and Wolf CR: Cross-talk between signalling pathways and the multidrug resistant protein MDR-1. Br J Cancer 85: 1175-1184, 2001.

3. Ganeshaguru K, Wickremasinghe RG, Jones DT, Gordon M, Hart SM, Virchis AE, Prentice HG, Hoffbrand AV, Man A, Champain K, Csermak K and Mehta AB: Actions of the selective protein kinase C inhibitor PKC412 on B-chronic lymphocytic leukemia cells in vitro. Haematologica 87: 167-176, 2002.

4. Castro AF, Horton JK, Vanoye CG and Altenberg GA: Mechanism of inhibition of P-glycoprotein-mediated drug transport by protein kinase C blockers. Biochem Pharmacol 58: 1723-1733, 1999.
5. Newman MJ, Rodarte JC, Benbatoul KD, Romano SJ, Zhang C, Krane S, Moran EJ, Uyeda RT, Dixon R, Guns ES and Mayer LD: Discovery and characterization of OC144-093, a novel inhibitor of P-glycoprotein-mediated multidrug resistance. Cancer Res 60: 2964-2972, 2000

6. Barancík M, Bohácová V, Sedlák J, Sulová Z and Breier A: LY294,002, a specific inhibitor of PI3K/Akt kinase pathway, antagonizes P-glycoprotein-mediated multidrug resistance. Eur J Pharm Sci 29: 426-434, 2006.

7. Kuo MT, Liu Z, Wei Y, Lin-Lee YC, Tatebe S, Mills GB and Unate H: Induction of human MDR 1 gene expression by 2acetylaminofluorene is mediated by effectors of the phosphoinositide 3-kinase pathway that activate NF-kappaB signaling. Oncogene 21: 1945-1954, 2002.

8. Chen B, Jin F, Lu P, Lu XL, Wang PP, Liu YP, Yao F and Wang SB: Effect of mitogen-activated protein kinase signal transduction pathway on multidrug resistance induced by vincristine in gastric cancer cell line MGC803. World J Gastroenterol 10: 795-799, 2004.

9. Choi BH, Kim CG, Lim Y, Shin SY and Lee YH: Curcumin down-regulates the multidrug-resistance $m d r l b$ gene by inhibiting the PI3K/Akt/NF kappa B pathway. Cancer Lett 259: 111-118, 2008.

10. Arboleda MJ, Lyons JF, Kabbinavar FF, et al: Overexpression of AKT2/protein kinase Bß leads to upregulation of $\beta 1$ integrins, increased invasion, and metastasis of human breast and ovarian cancer cells. Cancer Res 63: 196-206, 2003.

11. Strasser A, O'Connor L and Dixit VM: Apoptosis signaling. Annu Rev Biochem 69: 217-245, 2000.

12. Chatterjee D and Khoo KH: The surface glycopeptidolipids of mycobacteria: structures and biological properties. Cell Mol Life Sci 58: 2018-2042, 2001.

13. Holler N, Tardivel A, Kovacsovics-Bankowski M, et al: Two adjacent trimeric Fas ligands are required for Fas signaling and formation of a death-inducing signaling complex. Mol Cell Biol 23: $1428-1440,2003$.

14. Hymowitz SG, Christinger HW, Fuh G, et al: Triggering cell death: the crystal structure of Apo2L/TRAIL in a complex with death receptor 5. Mol Cell 4: 563-571, 1999.

15. Ashkenazi A and Dixit VM: Death receptors signaling and modulation. Science 281: 1305-1308, 1998.

16. Bhojani MS, Rossu BD and Rehemtulla A: TRAIL and antitumor responses. Cancer Biol Ther 2: S71-S78, 2003.

17. Eggert A, Grotzer MA, Zuzak TJ, et al: Resistance to tumor necrosis factor-related apoptosis-inducing ligand (TRAIL)induced apoptosis in neuroblastoma cells correlates with a loss of caspase-8 expression. Cancer Res 61: 1314-1319, 2001.

18. Shin EC, Seong YR, Kim CH, et al: Human hepatocellular carcinoma cells resist to TRAIL induced apoptosis, and the resistance is abolished by cisplatin. Exp Mol Med 34: 114-122, 2002.

19. Taniai M, Grambihler A, Higuchi H, et al: Mcl-1 mediates tumor necrosis factorrelated apoptosis-inducing ligand resistance in human cholangiocarcinoma cells. Cancer Res 64: 3517-3524, 2004.

20. Park SJ, Wu CH, Choi MR, Najafi F, Emami A and Safa AR: P-glycoprotein enhances TRAIL-triggered apoptosis in multidrug resistant cancer cells by interacting with the death receptor DR5. Biochem Pharmacol 72: 293-307, 2006.

21. Dida F, Li Y, Iwao A, Deguchi T, Azuma E and Komada Y: Resistance to TRAIL-induced apoptosis caused by constitutional phosphorylation of Akt and PTEN in acute lymphoblastic leukemia cells. Exp Hematol 36: 1343-1353, 2008.

22. Tabellini G, Tazzari PL, Bortul R, Evangelisti C, Billi AM, Grafone T, Martinelli G, Baccarani M and Martelli AM: Phosphoinositide 3-kinase/Akt inhibition increases arsenic trioxide-induced apoptosis of acute promyelocytic and T-cell leukaemias. Br J Haematol 130: 716-725, 2005.

23. Mantovani I, Cappellini A, Tazzari PL, Papa V, Cocco L and Martelli AM: Caspase-dependent cleavage of 170-kDa P-glycoprotein during apoptosis of human T-lymphoblastoid CEM cells. J Cell Physiol 207: 836-844, 2006.

24. Falà F, Blalock WL, Tazzari PL, Cappellini A, Chiarini F, Martinelli G, Tafuri A, McCubrey JA, Cocco L and Martelli AM: Proapoptotic activity and chemosensitizing effect of the novel Akt inhibitor (2S)-1-(1H-Indol-3-yl)-3-[5-(3-methyl-2Hindazol-5-yl)pyridin-3-yl]oxypropan2-amine (A443654) in T-cell acute lymphoblastic leukemia. Mol Pharmacol 74: 884-895, 2008.

25. Hao XS, Hao JH, Liu FT, Newland AC and Jia L: Potential mechanisms of leukemia cell resistance to TRAIL-induced apopotosis. Apoptosis 8: 601-607, 2003. 
26. Dupuis ML, Tombesi M, Sabatini M and Cianfriglia M: Differential effect of HIV-1 protease inhibitors on P-glycoprotein function in multidrug-resistant variants of the human $\mathrm{CD}^{+} \mathrm{T}$ lymphoblastoid CEM cell line. Chemotherapy 49: 8-16, 2003.

27. Di Maira G, Brustolon F, Bertacchini J, Tosoni K, Marmiroli S, Pinna LA and Ruzzene M: Pharmacological inhibition of protein kinase CK2 reverts the multidrug resistance phenotype of a CEM cell line characterized by high CK2 level. Oncogene 26: 6915-6926, 2007.

28. Cenni V, Maraldi NM, Ruggeri A, Secchiero P, Del Coco R, De Pol A, Cocco L and Marmiroli S: Sensitization of multidrug resistant human osteosarcoma cells to Apo2 ligand/TRAILinduced apoptosis by inhibition of the Akt/PKB kinase. Int $\mathrm{J}$ Oncol 25: 1599-1608, 2004.

29. Milani D, Zauli G, Rimondi E, et al: Tumour necrosis factorrelated apoptosis-inducing ligand sequentially activates prosurvival and pro-apoptotic pathways in SK-N-MC neuronal cells. J Neurochem 86: 126-135, 2003.

30. Calvo KR, Liotta LA and Petricoin EF: Clinical proteomics: from biomarker discovery and cell signaling profiles to individualized personal therapy. Biosci Rep 25: 107-125, 2005

31. Wulfkuhle JD, Speer R, Pierobon M, et al: Multiplexed cell signaling analysis of human breast cancer applications for personalized therapy. J Proteome Res 7: 1508-1517, 2008.

32. Petricoin EF III, Bichsel VE, Calvert VS, et al: Mapping molecular networks using proteomics: a vision for patient-tailored combination therapy. J Clin Oncol 23: 3614-3621, 2005.

33. Sarbassov DD, Guertin DA, Ali SM and Sabatini DM: Phosphorylation and regulation of Akt/PKB by the rictor-mTOR complex. Science 307: 1098-1101, 2005.

34. Vander Haar E, Lee SI, Bandhakavi S, Griffin TJ and Kim DH Insulin signalling to mTOR mediated by the Akt/PKB substrate PRAS40. Nat Cell Biol 9: 316-323, 2007.

35. Gao T, Furnari F and Newton AC: PHLPP: a phosphatase that directly dephosphorylates Akt, promotes apoptosis, and suppresses tumor growth. Mol Cell 18: 13-24, 2005.
36. Espina V, Liotta LA and Petricoin EF III: Reverse-phase protein microarrays for theranostics and patient tailored therapy. Methods Mol Biol 520: 89-105, 2009.

37. Kisucká J, Barancík M, Bohácová V and Breier A: Reversal effect of specific inhibitors of extracellular-signal regulated protein kinase pathway on P-glycoprotein mediated vincristine resistance of L1210 cells. Gen Physiol Biophys 20: 439-444, 2001.

38. Brognard J, Clark AS, Ni Y and Dennis PA: Akt/protein kinase B is constitutively active in non-small cell lung cancer cells and promotes cellular survival and resistance to chemotherapy and radiation. Cancer 61: 3986-3997, 2001.

39. Dutton A, Reynolds GM, Dawson CW, Young LS and Murray PG: Constitutive activation of phosphotidyl-inositide 3 kinase contributes to the survival of Hodgkin's lymphoma cells through a mechanism involving Akt kinase and mTOR. J Pathol 205: 498-506, 2005.

40. Vivanco I and Sawyers CL: The phosphatidylinositol 3-kinase Akt pathway in human cancer. Nat Rev Cancer 2: 489-501, 2002.

41. Gajate C and Mollinedo F: Cytoskeleton-mediated death receptor and ligand concentration in lipid rafts forms apoptosis-promoting clusters in cancer chemotherapy. J Biol Chem 280: 11641-1167, 2005.

42. Garofalo T, Giammarioli AM, Misasi R, et al: Lipid microdomains contribute to apoptosis-associated modifications of mitochondria in T cells. Cell Death Differ 12: 1378-1389, 2005.

43. Legembre P, Daburon S, Moreau P, et al: Amplification of Fasmediated apoptosis in type II cells via microdomain recruitment. Mol Cell Biol 25: 6811-6820, 2005.

44. Falschlehner C, Emmerich $\mathrm{CH}$, Gerlach B and Walczak H: TRAIL signalling: decisions between life and death. Int $\mathrm{J}$ Biochem Cell Biol 39: 1462-1475, 2007. 